\title{
2015 NIST Workshop on Analytical Transmission Scanning Electron Microscopy
}

\author{
Robert R. Keller ${ }^{1}$
}

${ }^{1 .}$ National Institute of Standards and Technology, Applied Chemicals and Materials Division, Boulder, CO, USA

The Workshop on Analytical Transmission Scanning Electron Microscopy (t-SEM) was held at the NIST Boulder Laboratories of the U. S. Department of Commerce September 15-16, 2015. Its intent was to assess present and future challenges and opportunities associated with the recent emergence of transmission approaches to performing scanning electron microscopy (SEM), historically a reflectionbased measurement platform for microscopic structure and chemistry of solids. Transmission techniques are particularly well-suited to characterization of materials that are confined to extremely small volumes, with dimensions in the range single to tens of nanometers, as well as materials composed of low atomic number elements such as carbon.

The following meeting objectives were targeted: (i) assess state of the art in t-SEM techniques, including diffraction, imaging, and spectroscopy; (ii) identify key areas of instrumental and analytical R \& D and standardization, to begin transformation of these novel techniques from curiosities into quantitative measurement methods; (iii) unify the global effort to accelerate development of analytical t-SEM to enable this new approach to material characterization to see widespread adoption. 41 participants attended, representing the electron microscope industry, analytical instrumentation industry, and users and developers within National laboratories and academia from around the world. The workshop included nine plenary lectures of length 45 minutes, each followed by 15 minutes of discussion, eleven poster preview presentations of length up to 5 minutes, and discussion of critical research issues in application-specific areas of diffraction, imaging, and spectroscopy.

At the time of the meeting, diffraction methods had by far seen the most development of the three tSEM analytical branches of diffraction, imaging, and spectroscopy. State of the art transmission electron diffraction in a commercial SEM takes the form of Kikuchi scattering of incident electrons that have reached the exit surface of a thin specimen positioned at or near horizontal close to the microscope pole piece [1,2]. A conventional electron backscatter diffraction (EBSD) system is typically used to detect those electrons, with patterns appearing extremely similar in contrast to those formed in the conventional reflection mode of EBSD. A horizontally-oriented EBSD phosphor, positioned immediately beneath a thin specimen, had just appeared in late 2015 [3], and showed promise for improved Kikuchi signal, as well as the formation of spot patterns. The conventional configuration has been used to produce crystallographic orientation maps with lateral spatial resolution of approximately 2 $\mathrm{nm}$ to $5 \mathrm{~nm}$. The conventionally-positioned EBSD configuration has also been used for measurement of isolated particles as small as $5 \mathrm{~nm}$ to $10 \mathrm{~nm}$. EBSD pattern sharpness algorithms applied to transmission electron diffraction patterns in the SEM have resulted in the creation of quasi-transmitted electron images that can reveal microstructural detail such as grain boundaries and defects like twins and dislocations, with contrast rivaling that found in conventional transmission electron microscope (TEM) bright-field images, and without diffraction contrast artifacts often found in conventional TEM imaging [2]. All major EBSD vendors have directed resources towards development of hardware and software for transmission electron diffraction in the SEM. These include specialized sample holders capable of securing TEM specimens, foils, and particles, and analytical programs that account for the substantially 
different sample positioning associated with transmission data collection.

Transmission electron imaging in the SEM is less mature. Over the past several years, commerciallyavailable scanning transmission electron microscopy (STEM) detectors have appeared on the market, resulting in the growing technology STEM-in-SEM. They have a wide range of capabilities. Electron conversion units as well as multi-diode systems now provide a modest level of control over acceptance angles of transmitted electrons, enabling the use of scattering mechanisms such as diffraction, massthickness, and atomic number contrast. The use of masks/apertures in conjunction with a STEM detector has resulted in significant improvements in the ability to control detector acceptance angles [4], with images under variable-angle bright-field, annular bright-field, and low-/medium-/high-angle annular dark-field conditions having been demonstrated. New developments in pixelated STEM detectors are also emerging, promising even greater control over acceptance angle [5].

Transmission spectroscopy methods in the SEM have seen the least amount of development. At the time of the workshop, there had been a few serious attempts to generate transmission energy-dispersive spectroscopy (EDS) data in an SEM. With the improved sensitivity of silicon drift detectors (SDDs), this remains a viable approach for attaining improved lateral spatial resolution over bulk measurements, although their implementation has not seen widespread attention. There was discussion of the ability to perform electron energy loss spectroscopy (EELS) in an SEM; at the time of the workshop, debate was on-going regarding advantages and disadvantages of a SEM approach to EELS, although products were under development.

The following were considered important areas to consider for advancing the transmission SEM measurement paradigm: SEM free-lens control; aberration correction; cleaner vacuum; more consideration to concurrent imaging modes, to include secondary electrons; careful consideration of effects of spurious X-ray formation in thin samples; improved sample stage stability and control; ability for scattering simulations to consider crystallography; studies of contrast formation associated with lowenergy electrons; improvements in terminology and definitions associated with newly-emerging transmission operational modes; more understanding of and control over beam damage; improvements to and developments of detectors; standards for thin film composition, mass-thickness, scanning distortions, beam damage, operational imaging modes, and sample preparation. A forthcoming NIST Special Publication will provide additional meeting detail.

References:

[1] R. R. Keller and R. H. Geiss, J. Microscopy 245 (2012), 245-251.

[2] P. W. Trimby, Ultramicroscopy 120 (2012), 16-24.

[3] J. J. Fundenberger et al., Ultramicroscopy 161 (2016), 17-22.

[4] J. Holm and R. R. Keller, Ultramicroscopy 167 (2016), 43-56.

[5] B. T. Jacobson et al., Proc. SPIE Vol. 9376 (2015), 93760K-1 - 93760K-13.

[6] This work is a contribution of the U.S. Department of Commerce and is not subject to copyright in the United States. 